\title{
Bipartite and Tripartite Entanglement of Truncated Harmonic Oscillator Coherent States via Beam Splitters
}

\author{
M. Daoud $^{a, 4 *}$, A. Jellal ${ }^{c, d, \oplus \oplus, \text { E.B. Choubabi }}{ }^{d, \phi \oplus}$ and E.H. El Kinani ${ }^{f \S}$ \\ ${ }^{a}$ Max Planck Institute for Physics of Complex Systems, D-01187 Dresden, Germany \\ ${ }^{b}$ Department of Physics, Faculty of Sciences, Ibn Zohr University, \\ PO Box 8106, 80006 Agadir, Morocco \\ ${ }^{c}$ Physics Department, College of Sciences, King Faisal University, \\ PO Box 380, Alahsa 31982, Saudi Arabia \\ ${ }^{d}$ Saudi Center for Theoretical Physics, Dhahran, Saudi Arabia \\ ${ }^{e}$ Theoretical Physics Group, Faculty of Sciences, Chouaïb Doukkali University, \\ PO Box 20, 24000 El Jadida, Morocco \\ ${ }^{f}$ Mathematics Department, Faculty of Science and Technical, Moulay Ismail University, \\ P.O. Box 509, Errachidia, Morocco
}

\begin{abstract}
We introduce a special class of truncated Weyl-Heisenberg algebra and discuss the corresponding Hilbertian and analytical representations. Subsequently, we study the effect of a quantum network of beam splitting on coherent states of this nonlinear class of harmonic oscillators. We particularly focus on quantum networks involving one and two beam splitters and examine the degree of bipartite as well as tripartite entanglement using the linear entropy.
\end{abstract}

\footnotetext{
*daoud@pks.mpg.de, m_daoud@hotmail.com

†ajellal@ictp.it, jellal@ucd.ma

${ }^{\ddagger}$ choubabi@gmail.com

§elkinani@gmail.com
} 


\section{Introduction}

Over the past few years, the entanglement of quantum systems was realized to be a valuable and crucial resource in quantum information processing. It allows for powerful new communication and computational tasks that are not possible classically. One may quote for instance quantum teleportation [1], superdense coding [2], quantum key distribution [3], telecloning [4], quantum cryptography [5] and quantum computation [6, 7]. Then it is not surprising then that over the last decade much efforts has been devoted to capture, quantify and assess the power of quantum entanglement. In this sense, many authors studied the development of a quantitative theory of entanglement and the definition of its basic measure (concurrence, entanglement of formation and linear entropy [8, 9, 10, 11]). Entangled quantum systems can exhibit correlations that cannot be explained on the basis of classical laws and the entanglement in a collection of states is clearly a signature of non-classicality [12].

In quantum theory, the states minimizing the quantum fluctuations, which are closest to the classical ones are the coherent states [13, 14]. This motivated the considerable interest in the entanglement of coherent states [15, 16, 17, 18]. The coherent state approach is not just a mathematical tool, but it my also helps to understand the entanglement for such states, which provide a bridge between quantum and classical worlds. It is also important to stress that in connection with quantum entanglement, many experimental results were obtained. In fact, the experimental generation and characterization of the entangled electromagnetic states can be achieved using type I or type II parametric down conversion [19]. Another experimentally accessible device, which can be used to generate optical entangled states, is the beam splitter [20, 21, 22]. In quantum optics the action of a beam splitter, which is essentially a coupling of two electromagnetic modes, can be represented by a unitary operator relating the input and the output states. In general, the output state is a superposition of the Fock states which is entangled, except the harmonic oscillator coherent states. Indeed, the harmonic oscillator coherent states does not exhibit entanglement when passed through one arm of 50:50 beam splitter while the second arm is left in the empty vacuum state [23]. In the same sense, the entanglement behavior of the $S U(2)$ spin coherent states, when passed through a beam splitter, was examined in [12]. Similar study was done in [24] for the $S U(1,1)$ coherent states.

On the other hand, the truncated harmonic oscillator was used by Pegg and Barnett [25] to define the phase states for the quantized single modes of the electromagnetic field. They suggested to truncate to some finite (but arbitrarily large) order the infinite-dimensional representation space of the oscillator algebra. This was done to get rid of the difficulty related to the infinite-dimensional character of the representation space of the Weyl-Heisenberg algebra, which constitutes a drawback in defining a phase operator in a consistent way [26, 27, 28]. Motivated by these investigations and in particular [25], we propose a refined version of the truncated oscillator algebra introduced by Pegg and Barnett. More precisely, we introduce a nonlinear class of Weyl-Heisenberg algebra and analyze its representations. We construct the associated coherent states using a suitable analytical realization and we study the degree of entanglement of such states when passed through a quantum network of beams splitters.

The outline of the paper is as follows. In section 2, we introduce a generalized Weyl-Heisenberg algebra. We discuss the corresponding Hilbertian representation and the analytical Bargmann realization. It is remarkable that the obtained analytical realization provides us with an over-complete set of 
states and then constitute a system of coherent states in the Klauder-Perelomov sense. In section 3 , we define the action of a quantum network of $k$ beam splitters. We show that this action leads to the $S U(k+1)$ coherent states labeled by complex variables and related to the reflection and transmission parameters of beam splitters. Based on this result, we investigate in detail the effect of one and two beam splitters on the generalized Weyl-Heisenberg algebra coherent states. We derive the linear entropy to study the bipartite entanglement degree of the output states. study the entanglement in a system of three particles. Concluding remarks close this paper.

\section{Weyl-Heisenberg algebra and Fock-Bargmann realization}

We start by introducing and discussing some interesting properties of a generalized version of the truncated harmonic oscillator, which involves a half positive integer $s$. In the limiting case when this parameter goes to the infinity, one recovers the usual harmonic oscillator with infinite dimensional Fock space. This provides us with another mathematical tool to deal with truncation of the ordinary harmonic oscillator that is different from one discussed in [25]. We mention that the idea of truncated Weyl-Heisenberg is mainly inspired by the polynomial deformation of Lie algebras introduced in [29, 30] and extensively discussed in the context of quantum algebras [31, 32, 33]. The truncated WeylHeisenberg algebra discussed here can be viewed as a special case of $f$-deformed oscillators introduced in [34, 35] which is relevant in the algebraic description of a large class of nonlinear quantum systems.

\subsection{Finite dimensional Weyl-Heisenberg algebra}

The basic ingredient that we shall use in what follows is the generalized Weyl-Heisenberg algebra characterized by a positive real parameter $(s>0)$. This algebra is generated by the set of operators $\left\{a^{+}, a^{-}, N, \mathbb{I}\right\}$ satisfying the relations

$$
\left[N, a^{-}\right]=-a^{-}, \quad\left[N, a^{+}\right]=+a^{+}, \quad\left[a^{-}, a^{+}\right]=\mathbb{I}-\frac{N}{s}
$$

where $N$ is the number operator and the element $\mathbb{I}$ commutes with all other operators. Clearly, when $s \rightarrow \infty$, end up with the ordinary Weyl-Heisenberg algebra. In this respect, the parameter $s$ can be regarded as a measure of the distortion of this algebra. For convenience, we assume that $2 s \in \mathbb{N}$. The generalized oscillator algebra can be naturally represented, on the Fock space $\mathcal{F}$ of the eigenstates of the number operator $N$, by

$$
N|n\rangle=n|n\rangle, \quad\langle n \mid m\rangle=\delta_{n m}, \quad n, m \in \mathbb{N}
$$

and the vacuum state satisfies $a^{-}|0\rangle=0$. We define the actions of the operators $a^{-}$and $a^{+}$on $\mathcal{F}$ as

$$
a^{-}|n\rangle=\sqrt{F(n)}|n-1\rangle, \quad a^{+}|n\rangle=\sqrt{F((n+1)}|n+1\rangle .
$$

Note that, $a^{+}$and $a^{-}$are mutually adjoint, namely $a^{+}=\left(a^{-}\right)^{\dagger}$ and $N$ is, in general, different from the product $a^{+} a^{-}$. The structure function $F(\cdot)$ is an analytic function with the properties $F(0)=0$ and $F(n)>0, n=1, \cdots, F(\cdot)$ is characteristic to the distortion or the truncation scheme and satisfies the following recursion formula

$$
F(n+1)-F(n)=1-\frac{n}{s}
$$


which gives by simple iteration the form

$$
F(n)=\frac{n}{2 s}(2 s+1-n) .
$$

Note that, the structure function obeys the condition

$$
F(2 s+1)=0
$$

and the creation-annihilation operators satisfy the nilpotency relations

$$
\left(a^{-}\right)^{2 s+1}=0, \quad\left(a^{+}\right)^{2 s+1}=0 .
$$

This means that the corresponding representation is $(2 s+1)$-dimensional. As evoked above the truncated oscillator algebra (11) constitutes a particular variant of the $f$-deformed oscillators [34, 35]. Indeed, one has

$$
a^{-}=b^{-} f(N), \quad a^{+}=f(N) b^{+}, \quad N=b^{+} b^{-},
$$

where the function $f(N)$, reflecting the distortion from the usual bosons, is given by

$$
f(N)=\sqrt{1-\frac{N-1}{2 s}} .
$$

The equation (8) is a Holstein-Primakoff realization of the algebra (1). Finally, we point out that for $s$ large, we have

$$
a^{ \pm} \sim b^{ \pm}
$$

traducing that the distorted algebra (11) coincides with the linear harmonic oscillator one. More importantly, the generalized Weyl-Heisenberg algebra provides us with a simply framework to deal with finite dimensional oscillator system. It is important to stress that this algebra is similar to one introduced in [36] in order to define the phase operator for nonlinear oscillators and to derive the associated temporally stable phase states. In this respect, the generalized Weyl-Heisenberg algebra can be viewed as a refined version of truncated harmonic oscillator.

Of course the problem of practical realization of the finite dimensional nonlinear quantum oscillator algebra arises. At this point one should emphasize that many exactly solvable systems enjoy the generalized Weyl-Heisenberg symmetry and are possessing finite dimensional representation space. One may quote for instance one dimensional quantum systems having finite discrete spectrums like ones evolving in the modified Pöschl-Teller [37] and Morse [38] potentials, see also [39]. Quantum systems described by a nonlinear Hamiltonian are familiar in the context of nonlinear quantum optics as for instance electromagnetic field propagating trough a nonlinear Kerr medium [40]. Indeed, the dynamical evolution of the electromagnetic field propagating in a Kerr medium can be described by the following Hamiltonian

$$
H_{\text {Kerr }}=b^{+} b^{-}-\kappa b^{+2} b^{-2}
$$

where $\kappa$ characterizes the Kerr nonlinearity. Setting $\kappa=\frac{1}{2 s}$, the Hamiltonian $H_{\text {Kerr }}$ coincides with the function structure $F(N)$ with $N \equiv b^{+} b^{-}$. It is also interesting to stress that the ladder operators $a^{+}$ and $a^{-}$can be related to Stokes operators introduced in [41, 42] in order to define a unitary operator 
representing the exponential of the phase difference between two modes of the electromagnetic field. This relation can be expressed as

$$
s_{+}=\sqrt{s} a_{+}, \quad s_{-}=\sqrt{s} a_{-}, \quad s_{3}=\frac{1}{2}(N-s)
$$

and one can simply verify that the Stokes generators $s_{+}, s_{-}$and $s_{3}$ satisfy the commutation relations of the $s u(2)$ algebra.

We close this subsection by noting that, in recent years, a special interest is devoted to the possibility of generating and manipulating systems, whose dynamics can be closed within a finite set of $n$-photon states. Such systems are very important for the implementation of models in the quantum information theory. The experimental devices leading to the finite-dimensional state generation are referred, in the literature, as linear [43, 44] or non-linear [45, 46] quantum scissors.

\subsection{Fock-Bargmann realization}

The second ingredient needed for our task is the coherent states associated with the above generalized algebra. A simply way to construct these states is to use the analytical Bargmann representation. Indeed, we realize the annihilation operator

$$
a^{-} \longrightarrow \frac{d}{d z}
$$

as derivation with respect to a complex variable $z$. The elements of the Fock space are realized as follows

$$
|n\rangle \longrightarrow C_{n} z^{n}
$$

Using the action of the operator $a^{-}$on the Fock space $\mathcal{F}$ given by (3) and the correspondence (11), one obtains the following recursion formula

$$
(n+1) C_{n+1}=\sqrt{F(n+1)} C_{n} .
$$

It follows that the coefficients $C_{n}$ are given by

$$
C_{n}=C_{0} \sqrt{\frac{2 s !}{(2 s)^{n} n !(2 s-n) !}} .
$$

To simplify, we set hereafter $C_{0}=1$. Having the expression of the coefficients $C_{n}$, one can determine the differential action of the creation operator $a^{+}$. Indeed, since the operator $N$ acts as

$$
N \longrightarrow z \frac{d}{d z}
$$

one can easily see, using the action of the generator $a^{+}$on the Fock space, that

$$
a^{+} \longrightarrow z-\frac{z^{2}}{2 s} \frac{d}{d z} \text {. }
$$

On the other hand, a general vector of $\mathcal{F}$

$$
|\phi\rangle=\sum_{n=0}^{2 s} \phi_{n}|n\rangle
$$


is represented in the Bargmann space as

$$
\phi(z)=\sum_{n=0}^{2 s} \phi_{n} C_{n} z^{n} .
$$

The inner product of two functions $\phi$ and $\phi^{\prime}$ is defined now as

$$
\left\langle\phi^{\prime} \mid \phi\right\rangle=\int d^{2} z \Sigma(z) \overline{\phi^{\prime}(z)} \phi(z)
$$

where the integration is carried out on the whole complex plane. The integration measure $\Sigma$, assumed to be isotropic, can be computed by choosing $|\phi\rangle=|n\rangle$ and $\left|\phi^{\prime}\right\rangle=\left|n^{\prime}\right\rangle$. Thus, one has to look for a solution of the following moment equation

$$
2 \pi \int_{0}^{+\infty} d \varrho \Sigma(\varrho) \varrho^{2 n+1}=\frac{(2 s)^{n} n !(2 s-n) !}{(2 s) !}
$$

where $\varrho=|z|$. To find the function satisfying the equation (18), we use Mellin transform technique to obtain

$$
\Sigma(\varrho)=\frac{2 s+1}{\pi}\left(1+\frac{\rho^{2}}{2 s}\right)^{-2 s-2} .
$$

At this stage, one can write the function $\phi(z)$ as the product of the state $|\phi\rangle$ with some ket $|\bar{z}\rangle$ labeled by the complex conjugate of the variable $z$. This is

$$
\phi(z)=\mathcal{N}\langle\bar{z} \mid \phi\rangle .
$$

Taking $|\phi\rangle=|n\rangle$, we obtain

$$
|z\rangle=\mathcal{N} \sum_{n=0}^{2 s} \sqrt{\frac{(2 s) !}{(2 s)^{n} n !(2 s-n) !}} z^{n}|n\rangle .
$$

The normalization constant for the the states (21) is given by

$$
\mathcal{N}=\left(1+\frac{|z|^{2}}{2 s}\right)^{-s}
$$

It is important to remark that the states (21) are coherent in the Klauder-Perelomov sense [13, 14]. Indeed, it is easy to see that the states $|z\rangle$ can be written as

$$
|z\rangle=\mathcal{N} \exp \left(z a^{+}\right)|0\rangle .
$$

They satisfy the over-completeness property

$$
\int d^{2} z \Sigma(|z|)|z\rangle\left\langle z\left|=\sum_{n=0}^{2 s}\right| n\right\rangle\langle n|
$$

where the measure $\Sigma(|z|)$ is given by (19). It should be noticed the states (21) are similar to nonlinear coherent states derived in [47]. They coincide with the standard Glauber coherent states for the usual harmonic oscillator at large $s$. 


\section{Quantum network of beam splitters}

In the recent years, the study of entangled states has revived interest in the beam splitter. This is due to the fact that this device offers a simple way to probe the quantum nature of electromagnetic field through simple experiments. As mentioned before, many authors studied the behavior of quantum states when passed through a beam splitter. This device is an optical element with two input ports and two output ports that, in some sense, governs the interaction of two harmonic oscillators. The input and output boson operators are related by a unitary transformation which is an element of the $S U(2)$ group. Recently, a quantum network of beam splitters was used to create multiparticle entangled states of continuous variables [48] and also multiparticle entangled coherent states [49]. Here we examine the entanglement of the coherent states (21) passing through a quantum network of beam splitters. Note that, a set of $k$ beam splitters can be experimentally used to generate $S U(k+1)$ coherent states labeled by the reflection and transmission parameters of the involved beam splitters.

\subsection{Generation of $S U(k+1)$ coherent states}

The most general transformation defining a quantum network of $k$ beam splitters is given by the unitary operator

$$
\mathcal{B}_{k}=\mathcal{B}_{k, k+1}\left(\theta_{k}\right) \mathcal{B}_{k-1, k}\left(\theta_{k-1}\right) \cdots \mathcal{B}_{1,2}\left(\theta_{1}\right)
$$

where the operators

$$
\mathcal{B}_{l, l+1}\left(\theta_{l}\right)=\exp \left[\frac{i}{2} \theta_{l}\left(b_{l}^{+} b_{l+1}^{-}+b_{l}^{-} b_{l+1}^{+}\right)\right]
$$

are the ordinary $S U(2)$ beam splitters with $l=1,2, \cdots, k$. The objects $b_{l}^{+}$and $b_{l}^{-}$are the usual harmonic oscillator ladder operators. The reflection and transmission coefficients

$$
t_{l}=\cos \frac{\theta_{l}}{2}, \quad r_{l}=\sin \frac{\theta_{l}}{2} .
$$

are defined in terms of the angles $\theta_{l}$. The operator $\mathcal{B}_{k}$ is actually acting on the states $\left|n_{1}, n_{2}, \cdots, n_{k}\right\rangle$ of the usual $k$-dimensional harmonic oscillator. If the input state is $\left|n_{1}, n_{2}, \cdots, n_{k}\right\rangle$, then the $\mathcal{B}_{k}$ action leads to the following Fock states superposition

$$
\mathcal{B}_{k}\left|n_{1}, n_{2}, \cdots, n_{k+1}\right\rangle=\sum_{m_{1}, m_{2}, \cdots, m_{k+1}} \mathcal{B}_{n_{1}, n_{2}, \cdots, n_{k+1}}^{m_{1}, m_{2}, \cdots, m_{k+1}}\left|m_{1}, m_{2}, \cdots, m_{k+1}\right\rangle
$$

and in general the output is a $(k+1)$-particle entangled state. On the other hand, the action of the unitary operator $\mathcal{B}_{k}$ on the state $\left|n_{1}, 0, \cdots, 0\right\rangle$ gives

$$
\begin{aligned}
\mathcal{B}_{k}\left|n_{1}, 0, \cdots, 0\right\rangle= & \mathcal{C} \sum_{n_{2}=0}^{n_{1}} \sum_{n_{3}=0}^{n_{2}} \cdots \sum_{n_{k+1}=0}^{n_{k}} \frac{\xi_{1}^{n_{2}} \xi_{2}^{n_{3}} \cdots \xi_{k}^{n_{k+1}} \sqrt{n_{1} !}}{\sqrt{\left(n_{1}-n_{2}\right) !\left(n_{2}-n_{3}\right) ! \cdots\left(n_{k}-n_{k+1}\right) ! n_{k+1} !}} \\
& \times\left|n_{1}-n_{2}, n_{2}-n_{3}, \cdots, n_{k+1}\right\rangle
\end{aligned}
$$

where the normalization constant reads as

$$
\mathcal{C}=\left(1+\left|\xi_{1}\right|^{2}+\left|\xi_{1}\right|^{2}\left|\xi_{2}\right|^{2} \cdots+\left|\xi_{1}\right|^{2}\left|\xi_{2}\right|^{2} \cdots\left|\xi_{k}\right|^{2}\right)^{-n_{1} / 2}
$$


and the new variables $\xi$

$$
\xi_{l}=i t_{l+1} \frac{r_{l}}{t_{l}} \quad \text { for }(l=1,2, \cdots, k-1), \quad \xi_{k}=i \frac{r_{k}}{t_{k}}
$$

are defined in terms of the reflection and transmission coefficients of the beam splitters constituting the network. Then, the output state (27) turns out to be the $S U(k+1)$ coherent state associated with the completely symmetric representation labeled by the integer $n_{1}$, see for instance [50] where similar notations were used. This means that a network of $k$ beam splitters can be used as experimental device to generate $S U(k+1)$ coherent states.

\subsection{Bipartite entanglement of truncated harmonic oscillator coherent states}

We start by investigating the effect of one beam splitter on the coherent states (21) associated with the generalized or truncated harmonic oscillator introduced in the first section. As discussed above, for $s$ large, the dimension of the generalized oscillator Fock space becomes infinite. In this case one can easily check that the coherent states (21) reduce to Glauber coherent states of the infinite dimensional harmonic oscillator. It has been proven that the Glauber states are the only pure states that when passed through one arm of the beam splitter, the output resultant states are disentangled [51]. In this respect, the main task here is to determine the behavior of the entanglement in terms of the Fock space dimension and to prove that the entanglement disappears when the Fock space dimension goes to infinity. For that end, we consider the situation where the coherent state (21) is injected into one port while no photon is injected into the other port.

Using the equation (27) for $k=1$, it is simply verified that the action of the beam splitter on the input state $|z, 0\rangle$ can be cast in the following form

$$
\mathcal{B}_{1,2}\left(\theta_{1}\right)|z, 0\rangle=\mathcal{N} \sum_{n=0}^{2 s} \sum_{q=0}^{2 s-n} \sqrt{\frac{(2 s) !}{(2 s-n-q) ! n ! q !}} t_{1}^{n}\left(i r_{1}\right)^{q} \frac{z^{q+n}}{(\sqrt{2 s})^{q+n}}|n, q\rangle
$$

where $\mathcal{N}$ is given by (22). To determine the degree of entanglement of the beam splitter output sates, we use the linear entropy which is the upper bound of the Von Neumann entropy [12]. This is

$$
S=1-\operatorname{Tr}\left(\rho_{1}^{2}\right)
$$

where $\rho_{1}=\operatorname{Tr}_{2} \rho_{12}$ is the reduced density operator obtained by tracing over the states of the second system. Using (30), the linear entropy is obtained as

$$
S=1-\sum_{n=0}^{2 s} \sum_{n^{\prime}=0}^{2 s} \sum_{q=0}^{\min \left(2 s-n, 2 s-n^{\prime}\right)} \sum_{q^{\prime}=0}^{\min \left(2 s-n, 2 s-n^{\prime}\right)} b\left(n, n^{\prime}, q\right) b\left(n^{\prime}, n, q^{\prime}\right)
$$

where

$$
b\left(n, n^{\prime}, q\right)=|\mathcal{N}|^{2}(2 s) ! \frac{|z|^{2 q}}{(2 s)^{q} q !} t^{n+n^{\prime}} r^{2 q} \frac{z^{n} \bar{z}^{n^{\prime}}}{(\sqrt{2 s})^{n+n^{\prime}}} \sqrt{\frac{1}{(2 s-n-q) !\left(2 s-n^{\prime}-q\right) ! n ! n^{\prime} !}} .
$$

At this stage, we have the necessary ingredients to study the behavior of the linear entropy $S$, which is rich. This is because $S$ is actually depending on three parameters: the truncation $s$ of the 
WeylHeisenberg algebra, the levels of excitation $|z|$ of the input coherent states and the reflection coefficient $r$ of the beam splitter device. We first plot the entanglement against $r^{2}$ for different finite dimensional Hilbert (the dimension is $2 s+1$ ) for coherent states having an excitation level $z=5$. The results are summarized in Figure 1:

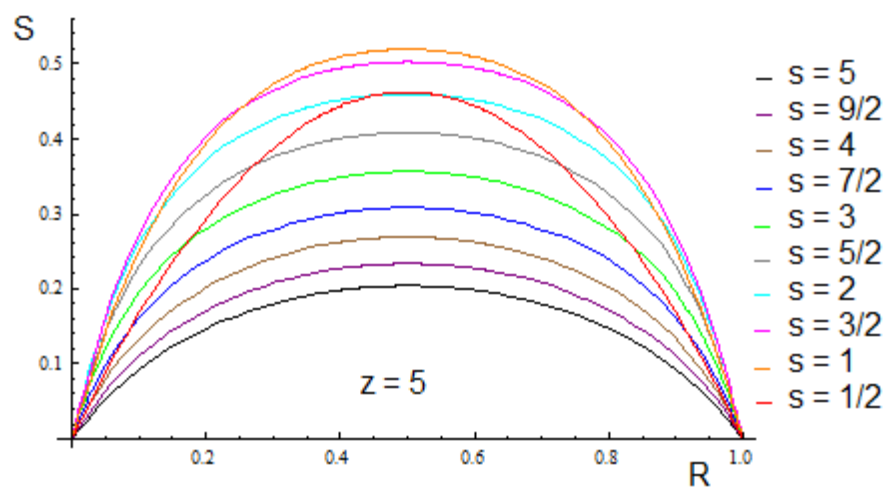

Figure 1: Linear entropy of coherent states as a function of the square of reflection coefficient $R=r^{2}$ for the level of excitation $z=5$.

It is clear that the maximum degree of entanglement is reach when one uses a 50:50 beam splitter (i.e. $r=1 / \sqrt{2}$ ) as expected. This is independent of the dimension of the Fock space. It is remarkable that for $r=1 / \sqrt{2}$, the linear entropy is maximal for $s=1$, which corresponds to qutrits system. The behavior of the entropy as function of the truncation level of the Weyl-Heisenberg algebra is represented in Figures 2. We consider separately coherent states with weak and strong excitation level passing throughout a 50:50 beam splitter.

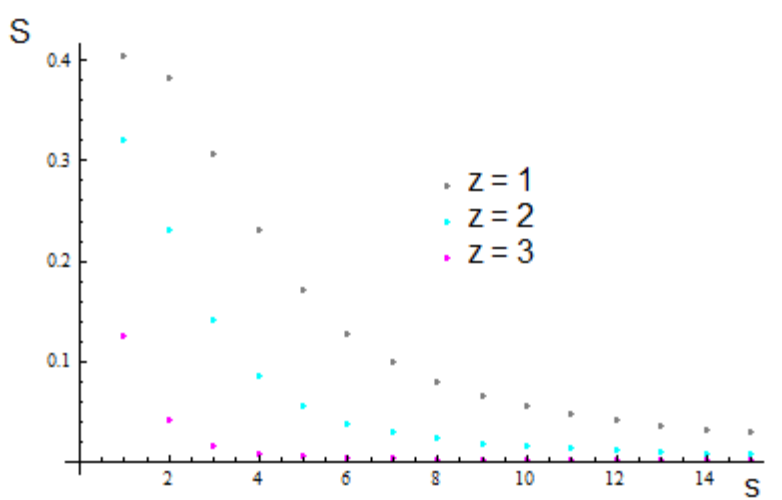

Figure 2.a: Linear entropy as a function of $s$ for weak coherent state excitation.

In Figure 2.a, it is clearly shown that for small values of the variable $z$, the linear entropy decreases quickly and goes to zero with increasing $s$. This agrees with the fact that for $s$ large, the linear entropy 
must vanishes as the coherent states (21) go to Glauber ones for ordinary harmonic oscillator, which does not exhibit entanglement after passing throughout a 50:50 beam splitter. In the case of stronger excitation, the linear entropy behaves differently as it can be seen from Figure 2.b.

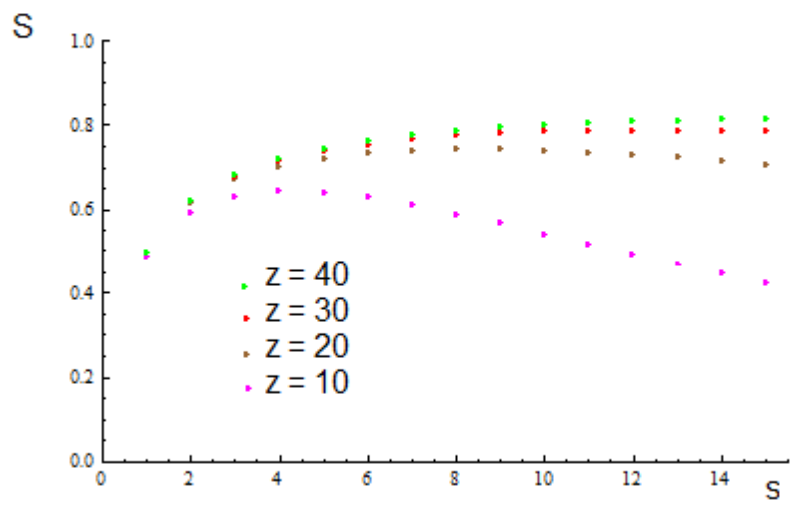

Figure 2.b: Linear entropy as a function of $s$ for strong coherent state excitation.

For instance, for $z=10$, the entropy undergoes an initial increase followed by a slower decrease. It follows that for strong excitation levels of the coherent states, the linear entropy will reach zero for very large value of $s$. To understand the behavior of the linear entropy as function of the excitation level of the coherent state $z$, we give the Figure 3:

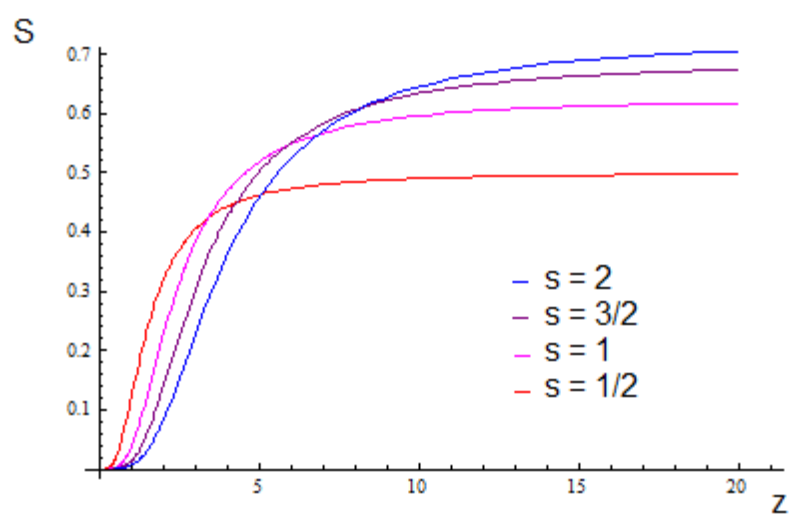

Figure 3: Linear entropy as a function of coherent state excitation.

In this Figure, we plot $S$ as function of $z$ for different values of $s$. It reflects that for weak excitation levels $z \leq 5$, the linear entropy increases rapidly and for higher level excitations it increases slowly. This explains why, for strong level excitation, the linear entropy does not go to zero for higher $s$ so quickly as in the case of systems with small number of states. The separability of highly excited 
coherent states, passing throughout a 50:50 beam splitter, occurs for quantum systems with sufficiently large number of states (the dimension of the corresponding Hilbert space is very large).

Two main features were observed through the analysis of this subsection. The first is that the entanglement of the output states depends heavily on $|z|$, more than $s$. For weak excitation levels $z$ of the coherent states, the linear entropy decreases quickly (Figure 2.a) as $s$ increases. This changes drastically for strong excitation levels and the entanglement initially increases with $s$ (Figure 2.b). The second interesting feature is that for large $|z|$, the entanglement, after an initial increase, decreases slowly as we vary $s$. This decline is very dependent on $|z|$ and is lower for larger $|z|$. It follows that to see coherent states (with s large) near zero entanglement, one would need a system of higher excitation levels. In our numerics we considered only $s$ such as $s \leq 15$, but we may see a significantly low resultant entanglement for large $s$ but with coherent states of extremely high excitation levels. This reflects the resistance of the bipartite entanglement of coherent states when passed through a beam splitter.

\subsection{Tripartite entanglement induced by two beam splitters}

To investigate the tripartite entanglement, we now consider the action of the unitary operator

$$
\mathcal{B}_{2}=\mathcal{B}_{2,3}\left(\theta_{2}\right) \mathcal{B}_{1,2}\left(\theta_{1}\right)
$$

on the coherent state (21). The operator $\mathcal{B}_{2}$ is obtained from (23) by setting $k=2$. This situation is interesting because it allows us to study bipartite as well three particle entanglement. The input states are of the form $|z ; 0 ; 0\rangle$ where the first mode is prepared in a coherent state $|z\rangle$ and the second and third modes are in their vacuum state. The action of $\mathcal{B}_{2}$ on the state $|z, 0,0\rangle$ can be evaluated as follows. Indedd, using (27), one verifies

$$
\mathcal{B}_{2}|n, 0,0\rangle=\sum_{p=0}^{n} \sqrt{\frac{n !}{p !(n-p) !}} t_{1}^{p}\left(i r_{1}\right)^{n-p} \sum_{p^{\prime}=0}^{n-p} \sqrt{\frac{(n-p) !}{p^{\prime} !\left(n-p-p^{\prime}\right) !}} t_{2}^{n-p}\left(i r_{2}\right)^{n-p-p^{\prime}}\left|p, n-p, n-p-p^{\prime}\right\rangle
$$

from which one obtains

$$
\left.\mathcal{B}_{2}|z, 0,0\rangle \equiv \mid \text { output }\right\rangle=\sum_{n=0}^{2 s} \sum_{p=0}^{n} \sum_{p^{\prime}=0}^{n-p} a\left(n, p, p^{\prime}\right)\left|p, p^{\prime}, n-p-p^{\prime}\right\rangle
$$

where

$$
a\left(n, p, p^{\prime}\right)=\mathcal{N} C_{n} z^{n} \sqrt{\frac{n !}{p !(n-p) !}} t_{1}^{p}\left(i r_{1}\right)^{n-p} \sqrt{\frac{(n-p) !}{p^{\prime} !\left(n-p-p^{\prime}\right) !}} t_{2}^{p^{\prime}}\left(i r_{2}\right)^{n-p-p^{\prime}} .
$$

The output state is a pure state of three qudits (1,2 and 3) system in the basis $\left\{\left|n_{1}, n_{2}, n_{3}\right\rangle\right\}$. Then, according to [52], for the pure state $\mid$ output $\rangle$ given by (36) defined on $\mathcal{F} \otimes \mathcal{F} \otimes \mathcal{F}(\operatorname{dim} \mathcal{F}=2 s+1)$, the concurrence has the form

$$
C(\mid \text { output }\rangle)=\sqrt{\frac{2 s+1}{12 s}\left[3-\left(\operatorname{Tr} \rho_{1}^{2}+\operatorname{Tr} \rho_{2}^{2}+\operatorname{Tr} \rho_{3}^{2}\right)\right]} .
$$

where

$$
\rho_{1}=\operatorname{Tr}_{2,3} \rho, \quad \rho_{2}=\operatorname{Tr}_{1,3} \rho, \quad \rho_{3}=\operatorname{Tr}_{1,2} \rho
$$


and $\rho=\mid$ output $\rangle\langle$ output $|$. This formula provides us with a measure of the tripartite entanglement of the coherent state (21) after passing through two beam splitters. We can interpret the right hand of the equation (38) as follows. If we regard the pair of oscillators (23) as a single subsystem, it makes sense to talk about the bipartite entanglement between the first oscillator system 1 and the pair (23). In this respect, one can treat 1 and (23) as two systems and use the linear entropy as measure of the degree of entanglement between them. In the same manner, one can define the bipartite entanglement between the subsystems 2 and (13) and the pair 3 and (12). The linear entropies in each case are given by

$$
S_{1}=1-\operatorname{Tr} \rho_{1}^{2}, \quad S_{2}=1-\operatorname{Tr} \rho_{2}^{2}, \quad S_{3}=1-\operatorname{Tr} \rho_{3}^{2} .
$$

It follows that the concurrence (38) can be expressed in terms of the sum of $S=S_{1}+S_{2}+S_{3}$. This reflects that the tripartite entanglement is related to the degree of bipartite entanglement between the different subcomponents of the system under consideration.

Figures 4, 5 and 6 present the behavior of the entropies $S_{1}, S_{2}$ and $S_{3}$ (40) in terms of the square of the reflection coefficients $r_{1}$ and $r_{2}$ of the beam splitters acting in the coherent state (21). We focus on qutrits system, i.e. $s=1$ and take the level of excitation of the input coherent state as $z=5$.

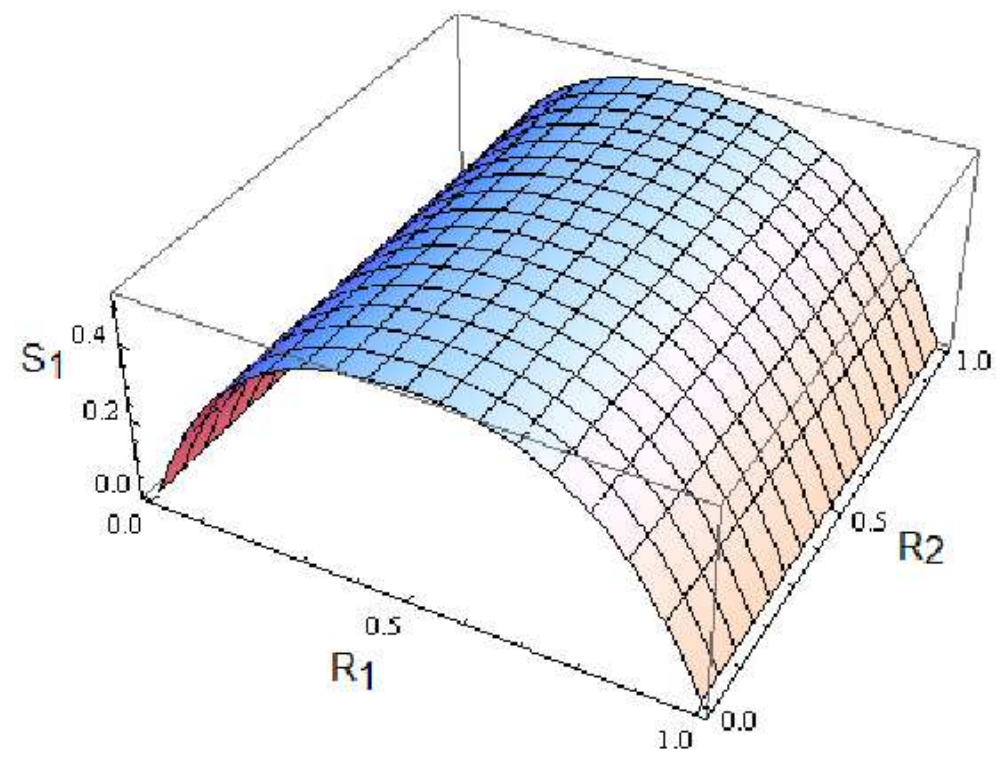

Figure 4: Linear entropy $S_{1}$ versus $R_{1}=r_{1}^{2}$ and $R_{2}=r_{2}^{2}$ for $(s=1, z=5)$.

The linear entropy $S_{1}$ measuring the degree of bipartite entanglement between the parts 1 and (23) viewed as a single subsystem is plotted in Figure 4. It is important to note that $S_{1}$ is maximal for $r_{1}=1 / \sqrt{2}$ and $r_{2}$-independent. This result can be easily shown analytically using the equations (36), (39) and (40). This is not the case for the entropy $S_{2}$ as it is shown by Figure 5: 


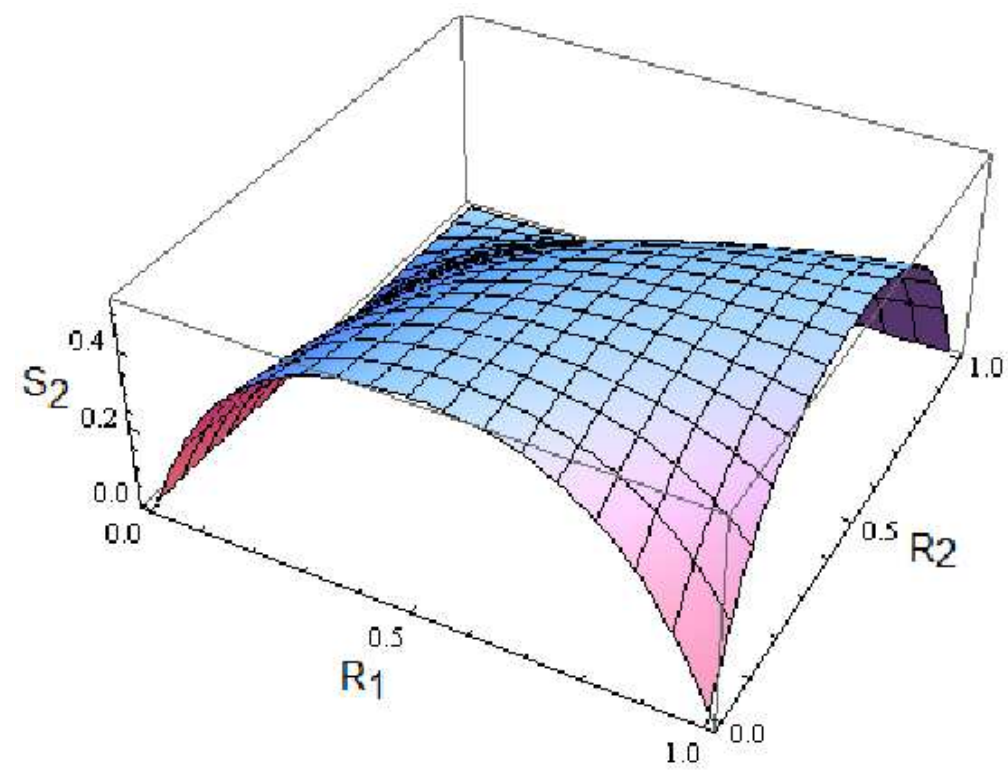

Figure 5: Linear entropy $S_{2}$ versus $R_{1}=r_{1}^{2}$ and $R_{2}=r_{2}^{2}$ for $(s=1, z=5)$.

The quantity $S_{2}$ is associated with the linear entropy measuring the degree of bipartite entanglement between the subsystems 2 and (13). The entropy $S_{2}$ is maximal for $\left(r_{1}=1 / \sqrt{2}, r_{2}=0\right)$ or $\left(r_{1}=\right.$ $1, r_{2}=1 / \sqrt{2}$ ) and vanishes for $r_{1}=0$ or $r_{2}=1$. Indeed, using the equation (36), one can simply check that the output state is a tensorial product of a wave function corresponding to the subsystem (13) and the vacuum $|0\rangle$ of the bosonic mode 2. Consequently, the linear entropy, associated with entanglement of the subsystems 2 and (13), vanishes in agreement with the results of Figure 5. Similarly, we plot in Figure 6 the linear entropy $S_{3}$ which gives the amount of entanglement between the subsystems (12) and 3 .

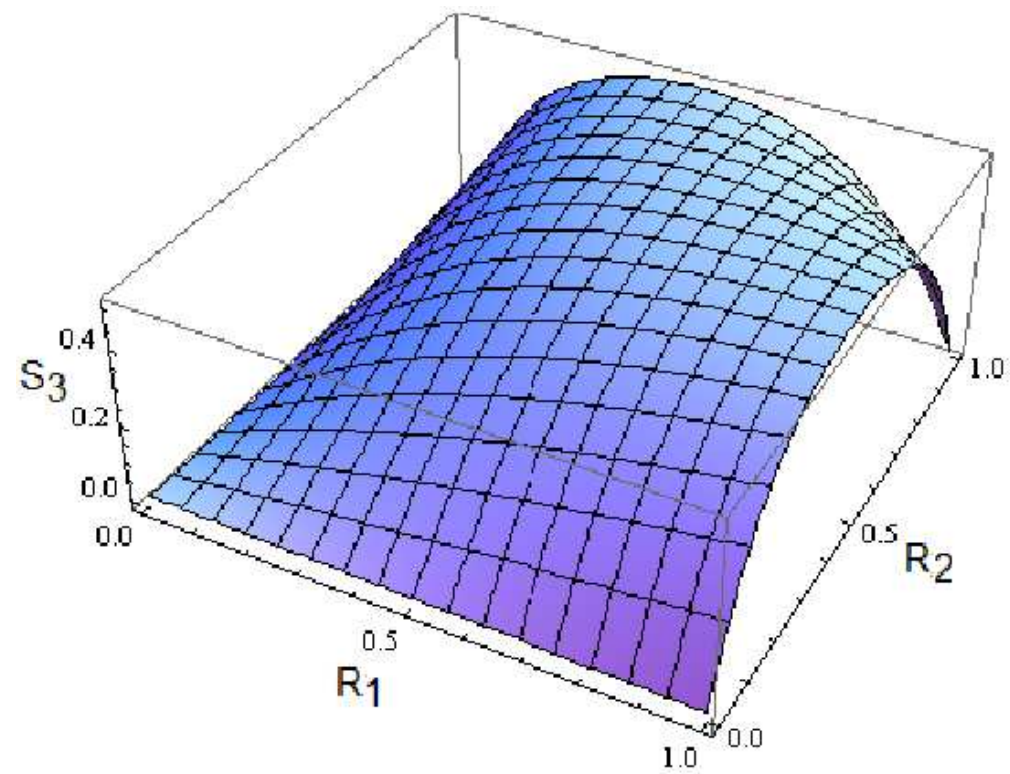

Figure 6: Linear entropy $S_{3}$ versus $R_{1}=r_{1}^{2}$ and $R_{2}=r_{2}^{2}$ for $(s=1, z=5)$.

The maximal value of the linear entropy $S_{3}$ is reached for $\left(r_{1}=1 / \sqrt{2}, r_{2}=1\right)$ or $\left(r_{1}=1, r_{2}=1 / \sqrt{2}\right)$ and vanishes for $r_{1}=0$ or $r_{2}=0$. This can be easily verified using the equation (36). Indeed, for 
$r_{1}=0$, the output state is separable because the action of the beam splitters leaves invariant the state $|z, 0,0\rangle$. Similarly, for $r_{2}=0$, the third part of the system, initially in the vacuum state, remains unchanged when passed throughout the beam splitters device and the output state is completely disentangled.

At this stage, one can use the linear entropies $S_{1}, S_{2}$ and $S_{3}$ to study the tripartite entanglement of the output state under consideration. In fact, from (38), it is easily seen that the square of the tripartite concurrence is proportional to the sum of the entropies $S_{1}, S_{2}$ and $S_{3}$. This quantity is plotted in Figure 7:

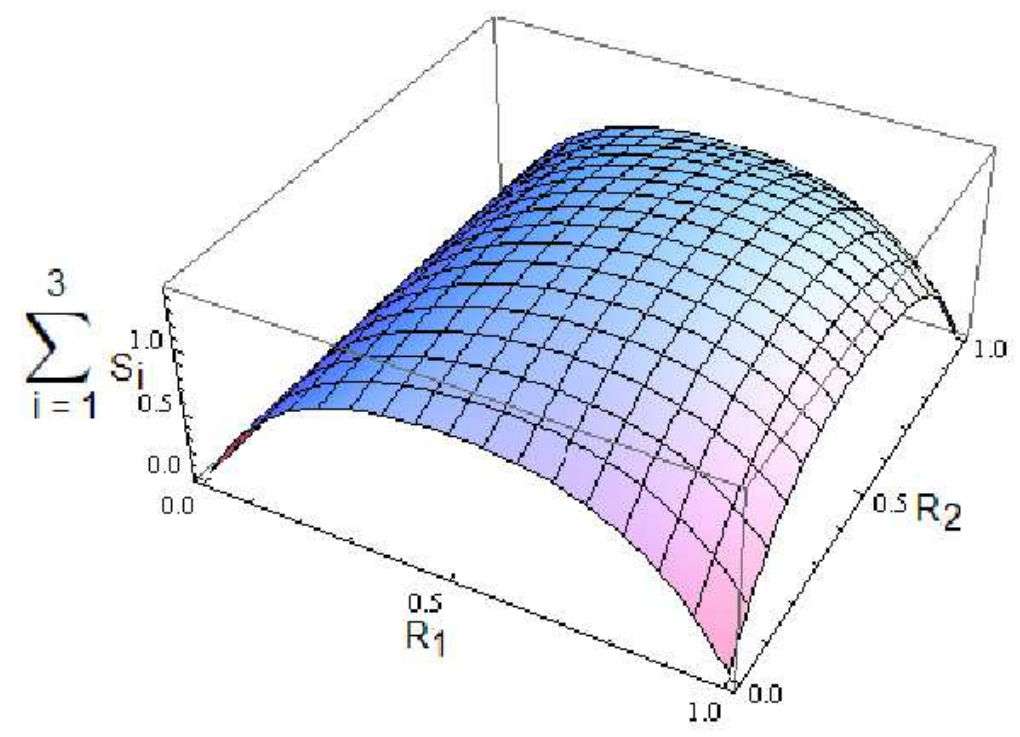

Figure 7: The total Linear entropy $S=S_{1}+S_{2}+S_{3}$ as functions of $R_{1}=r_{1}^{2}$ and $R_{1}=r_{2}^{2}$ for $(s=1, z=5)$.

We notice that for coherent states of a qutrit system $(s=1)$ with $z=5$, the maximal tripartite entanglement is obtained for $r_{1}=r_{2}=1 / \sqrt{2}$. It is interesting to mention that in the particular cases where $\left(r_{1}=0, r_{2}=0\right),\left(r_{1}=1, r_{2}=0\right),\left(r_{1}=0, r_{2}=1\right)$ and $\left(r_{1}=1, r_{2}=1\right)$, the output states are given by $|z, 0,0\rangle,|0, i z, 0\rangle,|z, 0,0\rangle$ and $|0,0,-z\rangle$, respectively. These states, derived from the equation (36), are clearly separable. This agrees with Figure 7 from which one can see that the entropy $S$ vanishes only in these particular four situations. In this subsection, we only considered qutrits. This study can be extended to any qudit system ( $s$ arbitrary). It should be noticed that for $s$ large and high excitation levels $z$, no entanglement can be generated at the output. This is essentially due, as we discussed in the previous subsection, to the fact that in this limit all the bipartite entanglement in the system vanishes and hence no tripartite entanglement can be generated. Indeed, for $s$ large the coherent states (21) reduces to Glauber ones. In this case, the action of two 50:50 beam splitters (i.e. $\left.i r_{1}=i r_{2}=\frac{1}{\sqrt{2}}\right)$, with a Glauber coherent states $\left.\mid z\right)$ incident in one port and a two particle vacuum $|0,0\rangle$ on the other ports, reduces to

$$
\mid z)|0\rangle|0\rangle \longrightarrow \mid z / \sqrt{2}) \mid i z / 2) \mid-z / 2)
$$


where the notation $\mid \alpha$ ) stands for the Glauber coherent states, such as

$$
\mid \alpha)=e^{-|\alpha|^{2} / 2} \sum_{n=0}^{\infty} \frac{\alpha^{n}}{n !}|n\rangle .
$$

This shows that in the limiting case $s \rightarrow \infty$, the resulting output state is disentangled. The results presented in this section can be extended in different directions to investigate many features of entangled coherent states passing throughout a quantum network of beam splitters.

\section{Conclusion}

Using the linear entropy as measure of the bipartite entanglement, we have investigated the bipartite entanglement of the coherent states of a truncated Weyl-Heisenberg algebra. This algebra constitutes an alternative way to describe quantized electromagnetic field with finite dimensional Hilbert spaces instead of the usual truncated harmonic oscillator introduced by Pegg and Barnett [25]. Subsequently, We have discussed the use of a quantum network of $k$ beam splitters as experimental device to generate easily the coherent states associated with $S U(k+1)$ algebra as well as the entangled photonic states.

We have focused on network involving one or two beam splitters. In the first case, this device is used to investigate the degree of entanglement of coherent state associated with the truncated harmonic oscillator. We have shown that, for weak level of excitation, the linear entropy goes faster to zero for $s$ increasing. For stronger excitation levels, the entropy decreases slowly as the Fock space dimension increases. The second case involves two beams splitters. In this case taking advantage from the relation of the tripartite concurrence and the partial linear entropies (see equation (38)), we have discussed the bipartite as well tripartite entanglement of a coherent states passing throughout a network of two beam splitters. Moreover, we have shown that the maximal tripartite entanglement is reached when the beam splitters are 50:50.

\section{Acknowledgments}

MD would like to express his thanks to Max Planck Institute for Physics of Complex Systems (DresdenGermany) where this work was done. AJ \& EBC thank the partial support of the Saudi Center for Theoretical Physics (SCTP) of Dhahran \& King Faisal University (KFU).

\section{References}

[1] C.H. Bennett, G. Brassard, C. Crépeau, R. Jozsa, A. Peres and W.K. Wootters, Phys. Rev. Lett. 70 (1993) 1895.

[2] C.H. Bennett and S.J. Wiesner, Phys. Rev. Lett. 69 (1992) 2881.

[3] A.K. Ekert, Phys. Rev. Lett. 67 (1991) 661.

[4] M. Murao, D. Jonathan, M.B. Plenio and V. Vedral, Phys. Rev. A 59 (1999) 156.

[5] C.A. Fuchs, Phys. Rev. Lett. 79 (1997) 1162. 
[6] R. Rausschendorf and H. Briegel, Quantum computing via measurements only, quant-ph/0010033.

[7] D. Gottesman and I. Chuang, Nature 402 (1999) 390.

[8] P. Rungta, V. Buzek, C.M. Caves, M. Hillery and G.J. Milburn, Phys. Rev. A 64 (2001) 042315.

[9] C.H. Bennett, D.P. DiVincenzo, J. Smolin and W.K. Wootters, Phys. Rev. A 54 (1996) 3824.

[10] W.K. Wootters, Phys. Rev. Lett. 80 (1998) 2245.

[11] V. Coffman, J. Kundu and W.K. Wootters, Phys. Rev. A 61 (2000) 052306.

[12] D. Markham and V. Vedral, Phys. Rev. A 67 (2003) 042113.

[13] J.R. Klauder and B.S. Skagerstam, Coherent states-Applications in Physics and Mathematical Physics (World Scientific, Singapore, 1985).

[14] A. Perelomov, Generalized Coherent States and their Applications,Texts and Monographs in Physics, (Spinger-Verlag, 1986).

[15] B.C. Sanders, Phys. Rev. A 45 (1992) 6811.

[16] C.C. Gerry, Phys. Rev. A 59 (1999) 4095.

[17] A. Luis, Phys. Rev. A 64 (2001) 054102.

[18] X. Wang, B.C. Sanders and S.H. Pan, J. Phys. A: Math. Gen. 33 (2000) 7451.

[19] P.G. Kwiat, S. Barraza-Lopez, A. Stefanov and N. Gisin, Nature 409 (2001) 1014.

[20] S.M. Tan, D.F. Walls and M.J. Collett, Phys. Rev. Lett. 66 (1991) 252.

[21] B.C. Sanders, K.S. Lee and M.S. Kim, Phys. Rev. A 52 (1995) 735.

[22] M.G.A. Paris, Phys. Rev. A 59 (1999) 1615.

[23] M.S. Kim, W. Son, V. Buzek and P.L. Knight, Phys. Rev. A 65 (2002) 032323.

[24] C.C. Gerry and A. Benmoussa, Phys. Rev. A 71 (2005) 062319.

[25] D.T. Pegg and S.M. Barnett, Phys. Rev. A 39 (1989) 1665.

[26] W.H. Louisell, Phys. Lett. 7 (1963) 60.

[27] L. Susskind and J. Glogower, Physics (U S) 49 (1964) 1.

[28] P. Carruthers and M.M. Nieto, Rev. Mod. Phys. 40 (1968) 411.

[29] P.W. Higgs, J. Phys. A: Math. Gen. 12 (1979) 309.

[30] E.K. Sklyanin, Funct. Anal. Appl. 16 (1982) 263. 
[31] M. Rocek, Phys. Lett. B 255 (1991) 554.

[32] D. Bonatsos, C. Daskaloyannis and G. Lalazissi, Phys. Rev. A 47 (1993) 3448.

[33] B. Abdesselam, J. Beckers, A. Chakrabarti and N. Debergh, J. Phys. A: Math. Gen. 29 (1996) 3075 .

[34] V.I. Man'ko, G. Marmo and F. Zaccaria , Proc. 4th Wigner Symp. Edited by N. M. Atakishiyev, T. H. Seligman and K B Wolf, (Singapore: World Scientific, 1996).

[35] V.I. Man'ko, G. Marmo, F. Zaccaria and E.C.G. Sudarshan, Phys. Scr. 55 (1997) 528.

[36] M. Daoud and M.R. Kibler, J. Phys. A: Math. Theor. 43 (2010) 115303.

[37] O. de los Santos-Sànchez and J. Récamier, J. Phys. A: Math. Theor. 44 (2011) 145307

[38] M. Daoud and D. Popov, Inter. J. Mod. Phys. B. 18 (2004) 325.

[39] M. Daoud and M. Kibler, J. Math. Phys. 47 (2006) 122108.

[40] A.B. Klimov, L.L. Sanchez-Soto, A. Navarro and E.C. Yustas, J. Mod. Opt. 49 (2002) 2211.

[41] A. Luis and L.L. Sanchez-Soto, Phys. Rev. A 48 (1993) 4702.

[42] A. Luis, Prog. Opt. 41 (2000) 421.

[43] D.T. Pegg, L.S. Phillips, and S.M. Barnett. Phys. Rev. Lett. 81 (1998) 1604.

[44] S.M. Barnett and D.T. Pegg, Phys. Rev. A 60 (1999) 4965.

[45] W. Leoǹski., Phys. Rev. A 55 (1997) 3874.

[46] W. Leoǹski, S Dyrting and R Tanaś, J. Mod. Opt. 44 (1997) 2105.

[47] J. Récamier and R. Jáuregui, J. Opt. B: Quantum and Semiclass. Opt. 5 (2003) S365.

[48] P. van Loock and S.L. Braunstein, Phys. Rev. Lett. 84 (2000) 3482.

[49] X. Wang, J. Phys. A: Math. Gen. 35 (2002) 165.

[50] M. Daoud, Phys. Lett. A 329 (2004) 318.

[51] Y. Aharonov, D. Falkoff, E. Lerner and H. Pendelton, Ann. Phys. 39 (1966) 498.

[52] Z. Ma and M. Bao, Phys. Rev. A 82 (2010) 034305. 